\title{
Questes
}

\section{Trouver la paix : éléments bibliographiques}

\section{Stéphanie Richard et Irène Strobbe}

\section{(2) OpenEdition}

\section{Journals}

Édition électronique

URL : http://journals.openedition.org/questes/1361

DOI : 10.4000/questes.1361

ISSN : 2109-9472

\section{Éditeur}

Les Amis de Questes

\section{Édition imprimée}

Date de publication : 25 septembre 2013

Pagination : 131-139

ISSN : 2102-7188

\section{Référence électronique}

Stéphanie Richard et Irène Strobbe, «Trouver la paix : éléments bibliographiques », Questes [En ligne], 26 | 2013, mis en ligne le 01 janvier 2014, consulté le 15 septembre 2020. URL : http:// journals.openedition.org/questes/1361 


\section{Bibliographie}

\section{Outils}

CARDINI, Franco, «Guerre et croisade », dans Dictionnaire raisonné de l'Occident médiéval, dir. Jacques Le GofF, Jean-Claude SchmitT, Paris, Fayard, 1999, p. 435-449.

GAUVARD, Claude, "Justice et paix », dans Dictionnaire raisonné de l'Occident médiéval, op. cit., p. 587-594.

Solere, Jean-Luc, « Paix », dans Dictionnaire du Moyen Âge, dir. Claude Gauvard, Alain De Libera, Michel ZinK, Paris, Presses Universitaires de France, 2002, p. 1034-1035.

\section{Ouvrages généraux sur la paix}

EBNER, Herwig, «Frieden im Mittelalter », Journal. Geschichte, juin-juillet 1989, p. 35-43.

Faire la guerre, faire la paix: approches sémantiques et ambiguïtés terminologiques, dir. Isabelle CHAVE, Paris, Comité des travaux historiques et scientifiques, édition électronique, 2012.

Les fondements de la paix, Des origines au début du XVIII siècle. Actes du colloque du Mémorial de Caen et du CRHQ (CNRS et Université) de Caen, dir. Pierre CHAUnU, Paris, PUF, 1993.

Frieden schaffen und sich verteidigen im Spätmittelalter/Faire la paix et se défendre à la fin du Moyen Âge, dir. Gisela NAEGLE, München, Oldenbourg Verlag, coll. « Pariser historische Studien », 2012.

Krieg und Frieden im Übergang vom Mittelalter zur Neuzeit: Theorie, Praxis, Bilder (Guerre et paix du Moyen Âge aux temps modernes: théories, pratiques, représentations), dir. Heinz DuchHARDT et Patrice VEIT, Mainz, P. von Zabern, 2000.

MACGuire, Brian Patrick, War and Peace in the Middle Ages, Copenhague, Reitzels Forlag, 1987.

Médiation, paix et guerre au Moyen Âge, dir. Michel SoT, Paris, Comité des travaux historiques et scientifiques, édition électronique, 2012. 
Miller, William Jan, Bloodtaking and Peacemaking. Feud, Law and Society in Saga Iceland, Chicago/London, Chicago University Press, 1990.

OHLER, Norbert, Krieg und Frieden im Mittelalter, Munich, Beck, 1997.

Pace e guerra nel basso Medioevo, éd. Centro Italiano di Studi sul Basso Medioevo, Spoleto, Fondazione Centro Italiano di Studi sull'Alto Medioevo, 2004.

La pace nel pensiero, nella politica, negli ideali del trecento, Todi, Accademia tudertina, coll. "Convengi del Centro di Studi sulla spiritulalità medievale », 15, 1975.

Paix de Dieu et guerre sainte en Languedoc au XIII siècle, Cahiers de Fanjeaux, Toulouse, Edouard Privat, 1969, 4.

Peace and Protection in the Middle Ages, dir. Tom B. LAMBERT, David Rollason, Durham/Toronto, Centre of Medieval and Renaissance Studies/Pontifical Institute of Medieval Studies, 2009.

La paix, Recueils de la société Jean Bodin pour l'histoire comparative des institutions, Bruxelles, Éditions de la Librairie encyclopédique, 1962, t. XIV et XV.

War and Peace in Ancient and Medieval History, dir. Philip De SouzA et John France, Cambridge/New York/Melbourne, Cambridge University Press, 2008.

\section{L’idée de paix}

Bonnaud-Delamare, Roger, L'idée de paix à l'époque carolingienne, Paris, Éditions Domat-Monchrestien, 1939.

CAROzZI, Claude, "La tripartition sociale et l'idée de paix au XI siècle », dans La guerre et la paix. Frontières et violences au Moyen Âge, Comité des travaux historiques et scientifiques. Actes du $101^{e}$ Congrès des Sociétés savantes, Lille, 1976.

Idees de Pau a l'Edat Mitjana, dir. Flocel SABAté i Curull, Maite Pedrol, Lleida, Pagès, 2010.

Lowe, Ben, Imagining Peace. A History of Early English Pacifist Ideas, 1340-1560, University Park, Pennsylvania State University Press, 1997.

RennA, Thomas, "The Idea of Peace in the West 600-1150 », Journal of Medieval Studies, 1980, 6, p. 143-167. 


\section{L'idée de paix dans la littérature}

Devaux, Jean, "Rhétorique et pacifisme chez Jean Molinet», dans Grands Rhétoriqueurs. Actes du colloque organisé à l'université de Paris-Sorbonne le 21 mars 1996, Paris, Presses de l’ENS, 1997.

Hagenlocher, Albrecht, Der " guote vride ». Idealer Friede in deutscher Literatur bis ins frühe 14. Jahrhundert, Berlin, W. De Gruyter, 1992.

VAn Hemelryck, Tania, «La femme et la paix. Un motif pacifique de la littérature française médiévale », Revue belge de philologie et d'histoire, 2006, vol. 84, n 2, p. 243-270.

YAeger, Robert F., «Pax Poetica: On the Pacifism of Chaucer and Gower », Studies in the Ages of Chaucer, 1987, 9, p. 87-21.

\section{Le règlement des conflits}

BALCH, Roscoe, "The Resigning of Quarrels : Conflict Resolution in the Thirteenth Century », Peace and Change, printemps 1978, vol. V, $\mathrm{n}^{\circ} 1$, p. 33-38.

CheYetTe, Frederik, «Suum cuique tribuere », French Historical Studies, 1970, 6, p. 287-299.

Conflitti, paci e vendette nell'Italia comunale, dir. Andrea ZorZI, Florence, Firenze University Press, 2009.

COUderC-BARRAUd, Hélène, La violence, l'ordre et la paix : Résoudre les conflits en Gascogne du XI au début du XIII siècle, Toulouse, Presses Universitaires du Mirail, coll. « Tempus », 2008.

Disputes and Settlements. Law and human relations in the West, dir. John Bossy, London/New York/Melbourne, Cambridge University Press, 1983.

GEARY, Patrick J., «Vivre en conflit dans une France sans État : typologie des mécanismes de règlement des conflits (1050-1200), Annales. Économies, Sociétés, Civilisations, $41^{\mathrm{e}}$ année, 1986, n 5 , p. 1107-1133.

L'infrajudiciaire du Moyen Âge à l'époque contemporaine, dir. Benoît GARNOT, Dijon, EUD Editions universitaires de Dijon, coll. «Publications de l'université de Bourgogne», Série du centre d'études historiques, 1996.

Lemesle, Bruno, Conflits et justice au Moyen Âge, Paris, Le Nœud Gordien, 2008. 
Powell, Edward, «Settlement of Disputes by arbitration in Fifteenth Century England », Law and History Review, 1984, 2, p. 21-43.

Le règlement des conflits au Moyen Âge, XXXI Congrès de la SHMESP (Angers, juin 2000), Paris, Publications de la Sorbonne, 2001.

Rosenthal, Joel T., "Feuds and Private Peace-making: A Fifteenth century Example », Nottingham Medieval Studies, 1970, 14, p. 84-90.

The Settlement of Disputes in Early Medieval Europe, dir. Wendy DAVIES et Paul Fouracre, Cambridge, Cambridge University Press, 1992.

White, Stephen D., «Pactum legem vincit et amor judicium. The Settlement of Disputes by Compromise in 11th Century France », The American Journal of Legal History, 1978, 22, p. 281-308.

White, Stephen D., «Feuding and peace-making in the Touraine around the year $1100 »$, Traditio, 1986, XLII, p. 195-263;

\section{Les acteurs de la paix}

Álvarez de Morales, Antonio, Las Hermandades, expresión del movimiento comunitario en España, Valladolid, Universidad de Valladolid, 1974.

Asenjo GonzÁlez, María, "Ciudades y Hermandades en la Corona de Castilla. Aproximación sociopolítica », Anuario de Estudios Medievales, 1997, 27, p. 103-146.

AUTRAND, Françoise, «Les artisans de paix face à l'État. La diplomatie pontificale et le conflit franco-anglais au XIV ${ }^{\mathrm{e}}$ siècle», dans Guerre et concurrence entre les États européens du XIV au XVIII siècle, dir. Philippe Contamine, PUF, Paris, 1998, p. 305-337.

CARL, Horst, Der Schwäbische Bund 1488-1534. Landfrieden und Genossenschaft im Übergang vom Spätmittelalter zur Reformation, Leinfelden-Echterdingen, DRW Verlag, 2000.

DÉPREZ, Eugène, « La conférence d'Avignon (1344). L'arbitrage pontifical entre la France et l'Angleterre », Essays in Medieval History presented to Thomas Frederick Tout [1925], dir. Andrew G. LiTTLE et Maurice POWICKE, Freeport/New York, Books for Libraries Press, 1967, p. 301-320.

GiBBOns, Rachel, «Les conciliatrices au bas Moyen Âge: Isabeau de Bavière et la guerre civile ", dans La guerre, la violence et les gens au Moyen Age, t. II, La violence et les gens, dir. Philippe CONTAMINE et Olivier GUYOTJEANNIN, Paris, CTHS, 1996, p. 23-33. 
Guillemain, Bernard, «Les tentatives pontificales de médiations dans le litige franco-anglais de Guyenne au XIV siècle », extrait du Bulletin philologique et historique (jusqu'à 1715) du Comité des travaux historiques et scientifiques, 1957, Paris, Imprimerie nationale, 1958.

«Il n'est tresor au monde que de paix». Hommes, lieux et instruments de pacification de l'Antiquité à nos jours, dir. Isabelle CLAUZEL, Boulognesur-Mer, Cercle d'études en Pays Boulonnais, 2007.

KAMP, Hermann, Friedensstifter und Vermittler im Mittelalter, Darmstadt, WBG, 2001.

Landfrieden. Anspruch und Wirklichkeit, dir. Arno Buschmann, Elmar WADLE, Paderborn, München, Schöningh, 2002.

MAGNOU-NorTIER, Élisabeth, «Les évêques et la paix dans l'espace franc ( $\mathrm{VI}^{\mathrm{e}}-\mathrm{XI}^{\mathrm{e}}$ siècles) », dans L'évêque dans l'histoire de l'Église. Actes de la septième rencontre d'histoire religieuse tenue à Fontevraud les 14 et 15 octobre 1983, Angers, Presses de l'Université d'Angers, 1984, p. 33-47.

MAHAUT, Marie-Claude, «Le rôle pacificateur du pape Benoît XII dans le conflit de la Castille avec le Portugal (1337-1340) », dans Actes du 101 Congrès national des sociétés savantes, Lille, 1976, Philologie et Histoire jusqu'à 1610, Paris, Bibliothèque nationale, 1978, p. 225-239.

MÉRINDOL (DE), Christian, «La femme et la paix dans la symbolique des décors à la fin de l'époque médiévale ", dans Regards croisés sur l'œuvre de Georges Duby, dir. Annie Bleton-Ruget, Marcelle PACAut et Michelle Rubellin, Lyon, Presses Universitaires de Lyon, 2000, p. 197-211.

Mollat, Guillaume, «Innocent VI et les tentatives de paix entre la France et l'Angleterre », Revue d'histoire ecclésiastique, 1909, X, p. 729-743.

OfFenstadt, Nicolas, "Les femmes et la paix à la fin du Moyen Âge : genre, discours, rites ", dans Le règlement des conflits au Moyen Âge, XXXIe Congrès de la SHMESP (Angers, juin 2000), Paris, Publications de la Sorbonne, 2001, p. 317-333.

Strohm, Paul, «Queens as intercessors », dans Hochon'Arrow. The Social Imagination of fourteenth-century texts, Princeton, Princeton University Press, 1992, coll. « Princeton paperbacks », p. 95-119.

Träger und Instrumentarien des Friedens im hohen und späten Mittelalter, dir. Johannes FrIED, Sigmaringen, Thorbecke, 1996.

WADLE, Elmar, Landfrieden, Strafe, Recht. Zwölf Studien zum Mittelalter, Berlin, Duncker \& Humblot, 2001. 


\section{Stratégies et discours sur la paix}

Althoff, Gerd, Spielregeln der Politik im Mittelalter. Kommunikation in Frieden und Fehde, Darmstadt, Primus Verlag, 1997.

Benham, Jenny, Peacemaking in the Middle Ages: principles and practice, Manchester/New York, Manchester University Press, 2011.

CARRÉ, Yannick, Le baiser sur la bouche au Moyen Âge. Rites, symboles, mentalités, $X I^{e}-X I V^{e}$ siècle, Paris, Le Léopard d'or, 1998.

Clayton, Dorothy J., "Peace Bonds and the Maintenance of Law and Order in Late Medieval England: The Example of Cheshire », Bulletin of the Institute of Historical Research, 1985, p. 133-150.

Crowder, C. M. D., "Peace and Justice around 1400 : a Sketch », dans Aspects of late medieval Government and Society. Essays presented to J. $R$. Lander, dir. John Gordon RowE, Toronto, University of Toronto Press, 1986, p. 53-81.

Frieden stiften. Vermittlung und Konfliktlösung vom Mittelalter bis heute, dir. Gerd Althoff, Darmstadt, WBG, 2011.

GAUVARD, Claude, "Cuisine et paix aux $\mathrm{XIV}^{\mathrm{e}}-\mathrm{XV}^{\mathrm{e}}$ siècles ", dans $L a$ sociabilité à table. Commensalité et convivialité à travers les âges, dir. Martin Aurell, Olivier Dumoulin, Françoise Thelamon, Rouen, Presses Universitaires de Rouen, 1992, p. 325-334.

Holdsworth, Christopher W., "Peacemaking in the Twelfth Century », Anglo-Norman Studies, 1998, 19, p. 1-18.

OfFEnStAdt, Nicolas, Faire la paix au Moyen Âge, Paris, Odile Jacob, 2007.

L'intercession du Moyen Âge à l'époque moderne. Autour d'une pratique sociale, dir. Jean-Marie MoEglin, Genève, Droz, 2004.

Paroles de paix en temps de guerre, dir. Sylvie CAUCANas, Rémy CAZALS et Nicolas OfFenstadT, Toulouse, Privat, 2006.

Peace and Negotiation: Strategies for Coexistence in the Middle Ages and the Renaissance, éd. Diane WolfTHAL, Turnhout, Brepols, coll. "Arizona Studies in the Middle Ages and the Renaissance », 4, 2000.

Petkov, Kiril, The Kiss of Peace, Ritual, Self and Society in the High and Late Medieval West, Leyde/Boston, Brill, 2003.

Prêcher la paix et discipliner la société, Italie, France, Angleterre (XIII $-X V^{e}$ siècles), dir. Rosa Maria DESSI, CNRS/Brepols, Turnhout, Collection d'études médiévales de Nice, 2005. 
VAN EICKels, Klaus, "Homagium and Amicitia: Rituals of Peace and their Significance in the Anglo-French Negotiations of the 12th Century », Francia, 1997, 24/1, p. 133-140.

VONES, Ludwig, «Un mode de résolution des conflits au bas Moyen Âge : le duel des princes ", dans La guerre, la violence et les gens au Moyen Âge, t. I, Guerre et violence, dir. Philippe CONTAMINE et Olivier GuYotJeAnNin, Paris, CTHS, 1996, p. 321-332.

\section{La paix de Dieu}

BARThÉlÉMY, Dominique, L'an mil et la paix de Dieu. La France chrétienne et féodale, Fayard, Paris, 1999.

Bonnaud-Delamare, Roger, "Fondements des institutions de paix au $\mathrm{XI}^{\mathrm{e}}$ siècle ", dans Mélanges d'histoire du Moyen Âge dédiés à la mémoire de Louis Halphen, Paris, Presses Universitaires de France, 1951, p. 21-26 .

Cowdrey, H. E. J., "The Peace and the Truth of God in the Eleventh Century », Past and Present, février 1970, 46, p. 42-67.

Gergen, Thomas, Pratique juridique de la paix et trêve de Dieu à partir du concile de Charroux (989-1250), Frankfurt/Main, Lang, 2004.

GraboIs, Aryeh, «De la trêve de Dieu à la paix du roi. Etude sur la transformation du mouvement de paix au XII siècle », dans Mélanges offerts à René Crozet, Poitiers, 1966, I, p. 585-596.

GrIGORE, Mihai-D., Ehre und Gesellschaft. Ehrkonstrukte und soziale Ordnungsvorstellungen am Beispiel der Gottesfrieden (10.-11. Jahrhundert), Darmstadt, WBG, 2009.

HofFmann, Hartmut, Gottesfriede und Treuga Dei, Stuttgart, Hiersemann, coll. « Schriften der Monumenta Germaniae Historica », 20, 1964.

La paix de Dieu, $X^{e}-X I$ siècle. Actes du colloque organisé en septembre 1987 par le Conseil général du département de la Haute-Loire, Le Puy, Conseil Général de Haute-Loire, 1988.

The Peace of God: Social Violence and Religious Response in France around the year 1000, dir. Thomas HEAD et Richard LANDES, Ithaca/London, Cornell University Press, 1992.

SÉMICHON, Ernest, La paix et la trêve de Dieu, Paris, Didier, 1857. 


\section{Paix et croisade}

DeLuZ, Christiane, «Croisade et paix en Europe au XIV ${ }^{\mathrm{e}}$ siècle. Le rôle du cardinal Hélie de Talleyrand », Cahiers de recherches médiévales, 1996, 1, p. 53-64.

FlORI, Jean, La guerre sainte. La formation de l'idée de croisade dans l'Occident chrétien, Paris, Aubier, 2001.

Mastnak, Tomaž, Crusading Peace. Christendom, The Muslim World, and Western Political Order, Berkeley/Los Angeles/London, University of California Press, 2002.

Powicke, Maurice R., «War as a Means to Peace : Some Late Medieval Themes ", dans Documenting the Past. Essays in Medieval History presented to G. P. Cuttino, dir. Jeffrey S. Hamilton et Patricia J. BradLEy, The Boydell Press, 1989, p. 217-224.

\section{Paix et diplomatie}

Allmand, Christopher, « The Anglo-French Negotiations, $1439 »$, Bulletin of The Institute of Historical Research, 1967, 40, p. 1-33.

Arras et la diplomatie européenne (XV ${ }^{e}-X V I^{e}$ siècles), dir. Denis ClaUZEL, Charles GIRY-Deloison, Christophe LeduC, Arras, Artois Presses Université, 1999.

AUTRAND, Françoise, «La paix impossible: les négociations francoanglaises à la fin du XIV ${ }^{\mathrm{e}}$ siècle », Annales de Bourgogne, 1996, t. 68, p. 11-22.

CHAPLAIS, Pierre, «Règlements des conflits internationaux franco-anglais au XIV ${ }^{\mathrm{e}}$ siècle (1293-1377) », Le Moyen Âge, 1951, LVII, p. 269-302.

DiCKInSOn, Jocelyne G., The Congress of Arras, 1435. A Study in medieval Diplomacy, Oxford, Clarendon Press, 1955.

DUPARC, Pierre, «La conclusion du traité de Troyes ", Revue historique de droit français et étranger, 1971, 1, p. 50-64.

Guerra y diplomacia en la Europa occidental 1280-1480, éd. Gobierno de Navarra, Pamplona, Fondo de Publicaciones del Gobierno de Navarra, 2005.

Petit-Dutaillis, Charles et Collier, Paul, « La diplomatie française et le traité de Brétigny », Le Moyen Âge, janvier-février 1897, I, p. 1-35. 


\section{Paix intérieure}

BARTHELEMY, Barthélemy, "Exorciser les démons de la vengeance en Flandre autour de $1100 »$, dans Au cloître et dans le monde. Femmes, hommes et sociétés ( $I X^{e}-X V^{e}$ siècle). Mélanges en l'honneur de Paulette L'Hermitte-Leclercq, éd. Patrick HenRIET et Anne-Marie Legras, Paris, Presses Universitaires de Paris-Sorbonne, 2000, p. 269-280.

Claustre, Julie, "Assurer la paix des "ménages". De la dette à la paix (Paris, XIV $-\mathrm{XV}^{\mathrm{e}}$ s.) », dans Frieden schaffen ..., op. cit., p. 199-214.

GAtier, Pierre-Louis, « Des femmes au désert? », dans Moines et religieux au Moyen Âge, éd. Jacques Berlioz, Paris, Seuil, coll. «Points histoire », 185, 1994, p. 171-186.

JONES, Eddie A., «Ceremonies of Enclosure : Rite, Rhetoric and Reality », dans Rhetoric of the Anchorhold: Space, Place, and Body within the Discourses of Enclosure, dir. Liz Herbert Mc Avoy, Cardiff, University of Wales Press, 2008, p. 37-46.

KING, Margot H., «The Desert Mothers: A Survey of the Feminine Anchoretic Tradition in Western Europe », Fourteenth Century English Mystics Newsletter, 9/1, mars 1983, p. 12-25.

L'HeRMite-LeClerCQ, Paulette, "Le reclus dans la ville au Bas Moyen Âge », Journal des Savants, 1988, n 3-4, p. 219-262.

L'HeRMiTe-LeCLERCQ, Paulette " La femme à la fenestrelle du reclusoir », dans La femme au Moyen Age. Actes du colloque de Maubeuge, 6-9 octobre 1998, dir. Michel Rouche et Jean Heuclin, Maubeuge/Paris, Touzot, 1990, p. 49-68.

Simons, Walter, Cities of Ladies. Beguine Communities in the Medieval Low Countries, 1200-1565, Philadelphie, University of Pennsylvania Press, 2001. 